\title{
Maximizing Vehicular Network Connectivity Through an Effective Placement of Road Side Units Using Voronoi Diagrams
}

\author{
Prithviraj Patil and Aniruddha Gokhale \\ *Vanderbilt University, Dept of EECS \\ Nashville, TN 37212, USA \\ Email: \{prithviraj.p.patil,a.gokhale\}@ vanderbilt.edu
}

\begin{abstract}
Vehicular Ad-hoc Networks (VANETs) are increasingly used to support critical services that improve traffic safety and alleviate traffic congestion. Developing VANET-based services and applications, however, is hindered due primarily to limited and often fluctuating communication capacity of VANETs that stem from the wireless and mobile nature of vehicle-tovehicle (V2V) communications. To address this limitation, RoadSide Units (RSU) have been proposed to complement V2V communication by providing event and data brokering capability in the form of Vehicle-to-Infrastructure (V2I) communications. This paper proposes a novel Voronoi network-based algorithm for the effective placement of RSU's which when deployed forms Voronoi networks in terms of the amount of delay incurred by data packets sent over the RSUs.
\end{abstract}

Index Terms-Vehicular networks; connectivity; placement.

\section{INTRODUCTION}

Vehicular Ad-hoc Networks (VANETs) are gaining significant attention due to their potential to support a variety of services of societal and environmental impact [3], [4], e.g. safety of passengers, reducing traffic congestion, driver assistance, etc. Despite the promise offered by VANETs, for these services to indeed be useful to the society and to scale to larger regions and vehicular traffic, VANETs must be able to overcome key challenges stemming from network connectivity bottlenecks caused in large part to the highly dynamic nature of the network, and bandwidth loss caused due to high and variable speeds of vehicles moving in different directions [2]. To overcome these challenges, it has been suggested to deploy road-side units (RSUs) as a means to provide a complementary communication capability in the form of a vehicle-toinfrastructure (V2I) network. Deployment of RSUs requires an upfront investment, and hence their deployment must be planned in accordance to present and projected traffic patterns and vehicular density. This paper proposes a novel algorithm for optimizing RSU usage by its placement according to traffic density, distance, and probability of $\mathrm{V} 2 \mathrm{~V}$ connection available on each road of the chosen region.

\section{Voronoi Network-BAsed RSU Placement}

This section presents our RSU placement algorithm that is based on the idea of a Voronoi Diagram [1]. The definition of Voronoi network (or Voronoi diagram) is quoted from [5] as follows: "Let $\mathrm{S}$ be a set of points in Euclidean space with all limit points contained in S. For every point $\mathrm{x}$ in the Euclidean space, there is one point of $\mathrm{S}$ closest to $\mathrm{x}$.

\section{A. Justification for Voronoi Diagrams for VANETs}

Let us assume that the RSUs are placed at the location of the generating point of the convex polygons of the Voronoi diagram that are formed for a given urban road network. In such a setup, the contours of the convex polygon that surrounds the RSU will be formed based on whatever criteria we choose to form the polygon. Due to the properties of Voronoi diagram, each vehicle will be now be covered by one RSU only, which also happens to be its nearest RSU from the perspective of delay incurred. This criterion maximizes the area covered by given RSUs while meeting the allowable delay bound for every vehicle since each vehicle based on its location is assigned to nearest RSU and no vehicle is left unassigned. If this criteria were aggregated with maximizing the number of vehicles served by the RSU, i.e., minimizing the packet losses, then by reconciling these two parameters in the formation of Voronoi diagram, we will have a solution for capacity planning for RSU placement problem in VANETs.

\section{B. Two-Stage Voronoi Diagram Algorithm for VANETs}

1) Stage I Algorithm: Stage I algorithm takes as input the maximum number of RSUs that are allowed by the city planners, a map of an urban area, mobility properties of traffic, and maximum tolerable delay for the service before its quality degrades, and a few other parameters. The algorithm starts by placing RSUs at some initial position either randomly or by spreading them evenly. If they are evenly spread out, the distance between any two RSUs is denoted $D_{\text {init }}$. To determine the density of vehicles in a region, our algorithm uses population density data available from census data and computes these parameters for every RSU.

We calculate extended range of RSU by assigning a single packet belonging to the chosen service to all the RSUs in the region and let the packet propagate from a given RSU towards other RSUs by leveraging the vehicles within its signal range (through V2I channel) as well as by hopping from one vehicle to another (through $\mathrm{V} 2 \mathrm{~V}$ channel).

Every packet will propagate a certain distance within a prescribed maximum duration $\left(d_{\max }\right)$ and may not necessarily 


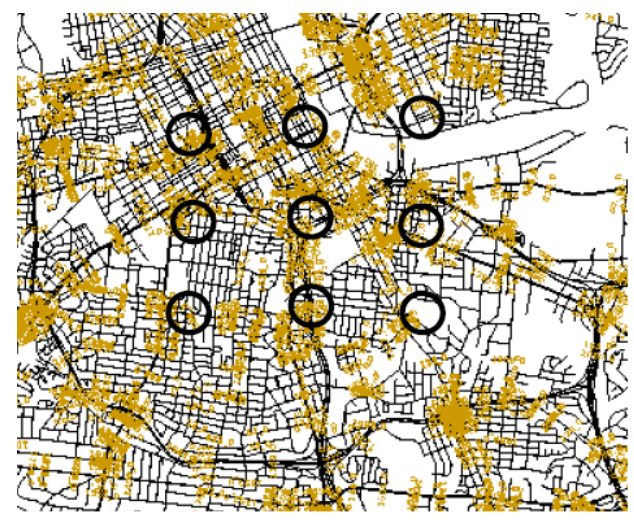

(a) Input to First Phase

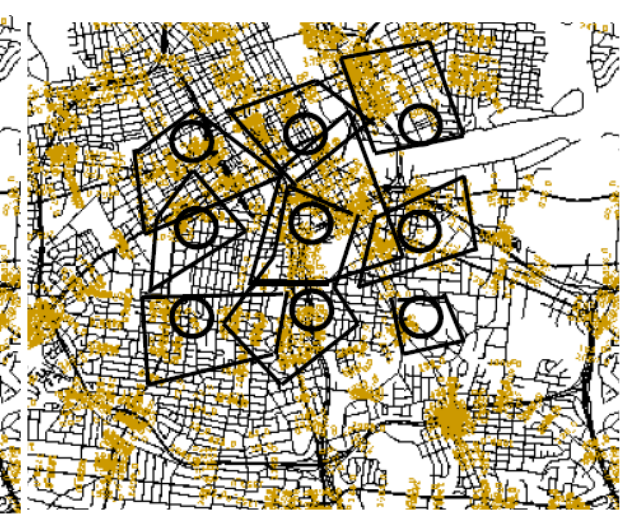

(b) Output of First Phase

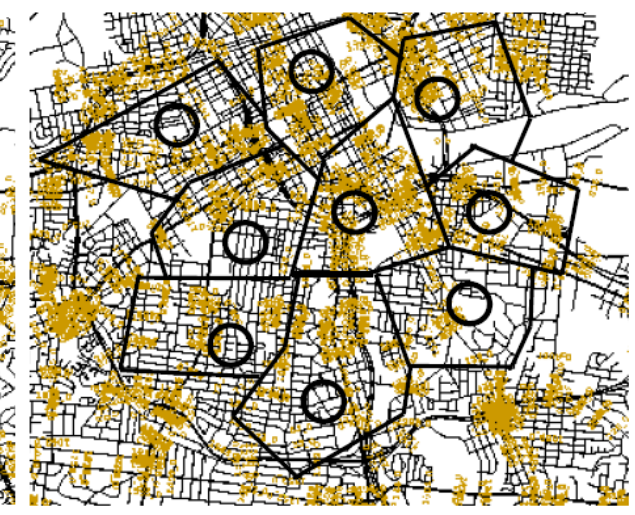

(c) Output of Second Phase

Fig. 1. Maximizing RSU Ranges Shown for 9 RSUs (Circle represents physical range; Polygon represents extended range)

reach the other RSU. The maximum farthest set of locations that a packet can travel given the mix of vehicles and their speeds along the routes they are allowed to take are computed once the packet delay bound is reached. A set of all such locations represents the extended range of a RSU. The extended range of RSU determines the contours of the polygon while the position of the RSU becomes the generating point. If the extended range of any two pairwise RSUs overlap, then the RSUs in the pair are deemed to be neighbors. Consequently, a neighborhood map of all RSUs for the entire region is computed. Also, each vehicle causes bandwidth loss because of its speed. To take this loss into consideration we compute, $N_{u}$ which is the portion of total vehicles which respect the delay bound despite bandwidth loss while relaying packet. $N_{u}$ determines level of overlap between two RSU ranges.

2) Stage II Algorithm: Stage II algorithm takes as input the number and position of initial RSUs with their neighbors and $N_{u}$. It then iterates for each RSU by visiting its neighbors over all possible routes and tries to relocate itself at a place where it will witness maximum $N_{u}$. Since every RSU will try to maximize its $N_{u}$ (at the cost of neighbor), at end of the iteration, $N_{u}$ will be shared equally among all RSUs. Also this will remove any unattended spaces since in order to maximize its $N_{u}$, a RSU will try to incorporate such areas in its extended range. Fig 1 shows the input and output for each algorithm stage for a map of urban area with 9 initial RSUs.

\section{EXPERIMENTAL EVALUATION}

We compare our approach against other two approaches for placement of RSUs namely even placement and busiest junction placement using three different metrics of evaluation. In the first metric, we study the extended range of the RSU. The larger the range, the larger is the area that can be covered without impacting reliability of communications. The second metric evaluates packet loss which is a direct indicator of how reliable is the communication. The third metric evaluates average packet delay, which is an indicator of assurance of quality of service. Initial results show that our approach perform better in all the three metrics. We are in the process of obtaining more results for evaluation to validate the performance of our algorithmic approach.

\section{Conclusion And Future Work}

We presented the algorithm that adjusts the placement of RSU in accordance to the traffic data populated from census data of Tiger maps of US census bureau. In doing so, it benefits from the traffic patterns and maximizes the normal range of RSU to an extended range. This in turn optimizes the number of RSUs required to achieve a specific quality of service of connectivity. As a future work, we will evaluate our algorithm for various quality of service requirements for each individual packet depending on the application.

\section{ACKNOWLEDGEMENTS}

"This work was supported in part by the National Science Foundation NSF SHF/CNS Award CNS 0915976, and NSF RAPID Awards 1047792 and 1047753. Any opinions, findings, and conclusions or recommendations expressed in this material are those of the author(s) and do not necessarily reflect the views of the National Science Foundation." "This work was supported in part by the National Science Foundation NSF SHF/CNS Award CNS 0915976, and NSF RAPID Awards 1047792 and 1047753. Any opinions, findings, and conclusions or recommendations expressed in this material are those of the author(s) and do not necessarily reflect the views of the National Science Foundation."

\section{REFERENCES}

[1] Franz Aurenhammer. Voronoi diagrams a survey of a fundamental geometric data structure. ACM Comput. Surv., 23:345-405, September 1991.

[2] Dirk Helbing. Traffic and related self-driven many-particle systems. Rev. Mod. Phys., 73:1067-1141, Dec 2001

[3] T. Mangel and H. Hartenstein. An analysis of data traffic in cellular networks caused by inter-vehicle communication at intersections. In Intelligent Vehicles Symposium (IV), 2011 IEEE, pages $473-478$, june 2011.

[4] Sok-Lan Sou. A power-saving model for roadside unit deployment in vehicular networks. Communications Letters, IEEE, 14(7):623 -625, july 2010.

[5] Wikipedia. Voronoi diagram, November 2011. 